\title{
N1-Arylation of 1,4-Benzodiazepine-2-ones with Diaryliodonium Salts
}

\author{
Raysa Khan ${ }^{a}$ \\ Robert Felix ${ }^{b}$ \\ Paul D. Kemmittc \\ Simon J. Coles ${ }^{\text {d }}$ \\ Graham J. Tizzardd \\ John Spencer*a (iD) \\ a Department of Chemistry, School of Life Sciences, University of Sussex, Falmer, \\ BN19QJ, UK \\ j.spencer@sussex.ac.uk \\ b Tocris, Bio Techne, The Watkins Building, Atlantic Road, Bristol, BS11 9QD, UK \\ ' IMED Oncology, AstraZeneca, 310 Cambridge Science Park, Milton Road, Cambridge \\ CB4 OWG, UK \\ d UK National Crystallography Service, Chemistry, University of Southampton, High- \\ field, Southampton, SO17 1BJ, UK
}

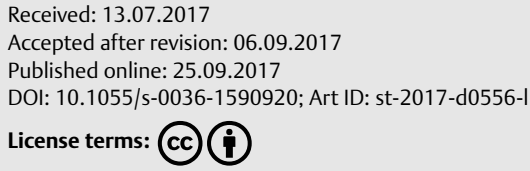

Abstract A library of N1-arylated 5-phenyl-1,3-dihydro-2H-1,4-benzodiazepin-2-ones has been synthesized starting with unsymmetrical diaryliodonium salts using aqueous ammonia as a base. This can also be applied to a similar 1,3,4-benzotriazepin-2-one derivative.

Key words benzodiazepines, $\mathrm{N}$-arylation, iodonium salt, privileged scaffold, benzotriazepine

Compounds containing a 1,4-benzodiazepine scaffold are often termed as 'privileged structures' and are of significant interest to organic and medicinal chemists. ${ }^{1-18}$ Many bioactive 1,4- benzodiazepines include $N$-arylated benzodiazepines; for example, the benzodiazepine derivative $\mathbf{A}$ (Figure 1) is a bradykinin antagonist ${ }^{19}$ and the related benzotriazepine $\mathbf{B}$ is an antagonist at the parathyroid hormone (PTH)-1 receptor. ${ }^{20}$ Typically $N$-arylated benzodiazepines can be prepared by transition-metal- catalysed couplings, often with copper, with various arylating agents. Generally, the reaction scope is limited with these routes and often requires high temperatures and strong bases. ${ }^{19,21-23}$
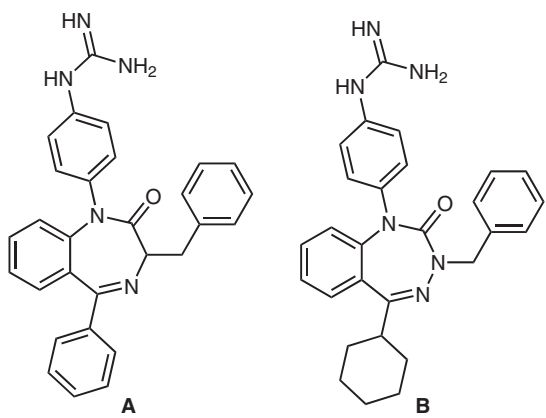

Figure 1 Bioactive $N$-arylated Benzodiazepine and Benzotriazepine

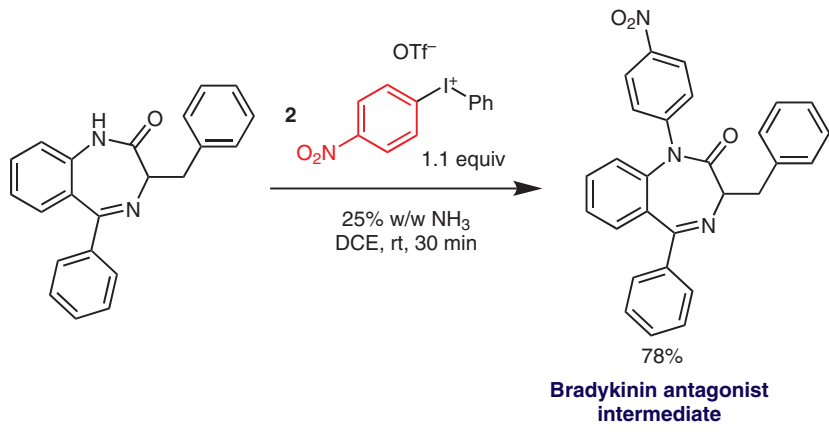

Being able to generate libraries of diverse analogues, in this case by adding $N$-functionality to a privileged core unit, using mild and efficient methodologies, can substantially improve SAR studies (structure-activity relationship) and optimise the drug development process potentially repurposing privileged scaffolds for new biological targets. ${ }^{24,25}$

We have an active interest in benzodiazepines ${ }^{26,27}$ and recently reported a method to functionalise 5-phenyl-1,3dihydro-2H-1,4-benzodiazepin-2-ones via a late-stage palladacycle assisted ortho $\mathrm{C}-\mathrm{H}$ activation protocol. ${ }^{28,29}$ Herein we present our approach to generate a series of $N 1$-arylated 1,4-benzodiazepines using diaryliodonium salts. The latter react with nucleophiles in the absence of transition-metal catalysts and are commonly used in organic synthesis as electrophilic reagents. ${ }^{30-35}$

Novak et al. recently reported a protocol for the $\mathrm{N}$-arylation of pyrazoles. ${ }^{36} \mathrm{~A}$ quick screen of conditions, adapting this protocol using diaryliodonium salts with weak bases under mild conditions, showed that it was indeed possible to perform similar arylations on the 1,4-benzodiazepine system. Upon initial screening of a number of solvents, 1,2dichloroethane (DCE) was found to give the best results (Table 1, entry 2). Solvents such as polypropylene glycol (PEG) and acetic acid $(\mathrm{AcOH})$ gave poor yields. Similar results were observed on pyrazoles by Novak et al. where aprotic solvents, immiscible in water, produced the best results.

A number of bases were tested subsequently and both $\mathrm{NH}_{3}(25 \% \mathrm{w} / \mathrm{w})$ and $\mathrm{NaOH}$ (sat. aq.) gave similar and the best results (Table 2, entries 1, 2).

Hence, optimal conditions appeared to use $\mathrm{NH}_{3}$ (aq.), DCE at room temperature for $30 \mathrm{~min}$. Next, a series of functionalized 1,4-benzodiazepines was $\mathrm{N}$-arylated using (4-nitrophenyl)phenyliodonium triflate in good to excellent yields (Scheme 1). Generally, in transition-metal-free processes unsymmetrical diaryliodonium salts give a mixture of products where both groups are transferred and the transfer of more sterically hindered and electron-with- 
Table 1 Optimization of N-Arylation of 1,4-Benzodiazepines - Solvent Effects

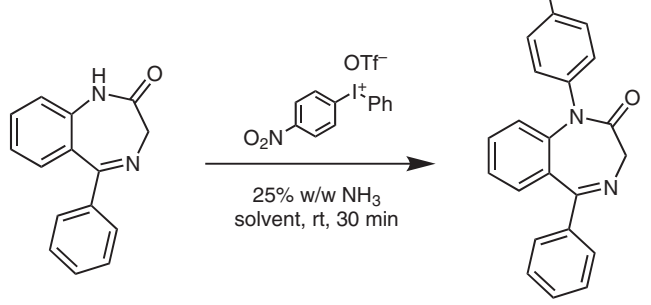

\begin{tabular}{lll}
\hline Entry & Solvent & Conversion (\%) $^{\mathrm{a}}$ \\
\hline 1 & toluene & 95 \\
2 & DCE & 99 \\
3 & PEC & - \\
4 & $\mathrm{AcOH}$ & - \\
5 & $\mathrm{CHCl}_{3}$ & 85 \\
\hline
\end{tabular}

a LC-MS conversion.

drawing groups is preferable. ${ }^{34}$ However, in this case (Scheme 1) only the nitrophenyl group was transferred. We were able to $N$-arylate quite sterically hindered benzodiazepines such as $\mathbf{3 e}$, 3f, and $\mathbf{3 g}$. Of note, $\mathbf{3 e}$ is a key intermedi-
Table 2 Optimization of N-Arylation of 1,4-Benzodiazepines - Base Effects

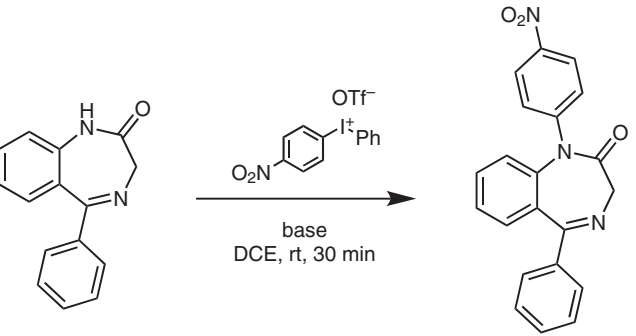

\begin{tabular}{lll}
\hline Entry & Base & Conversion (\%) \\
\hline 1 & $\mathrm{NaOH}($ sat. aq. $)$ & 99 \\
2 & $\mathrm{NH}_{3}(25 \% \mathrm{w} / \mathrm{w})$ & 99 \\
3 & $\mathrm{~K}_{2} \mathrm{CO}_{3}$ & 80 \\
4 & $\mathrm{NaH}$ & - \\
\hline a LC-MS conversion. &
\end{tabular}

ate towards $\mathbf{A}$. We were also pleased to be able to conduct $\mathrm{N}$-arylation on a previously ortho-arylated hindered benzodiazepine, $\mathbf{3 h}$, in good yield, whose structure was also confirmed by X-ray crystallography. Such molecules may be useful precursors to, e.g., $\alpha$-helical mimetics in medicinal chemistry. ${ }^{37,38}$<smiles>[R]C1=NC([R])C(=O)Nc2ccc([X])cc21</smiles>

1<smiles>CC1=NCC(=O)N(c2ccc([N+](=O)[O-])cc2)c2ccccc21</smiles>

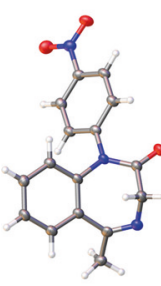

3a<smiles>O=C1C(Cc2ccccc2)N=C(c2ccccc2)c2cc(Cl)ccc2N1c1ccc([N+](=O)[O-])cc1</smiles>

3f; $75 \%$

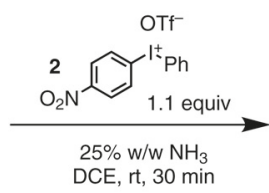<smiles>CCC(C)C1=NCC(=O)N(c2ccc([N+](=O)[O-])cc2)c2ccccc21</smiles>

3b; $99 \%$<smiles>O=C1C(Cc2ccccc2)N=C(c2ccccn2)c2ccccc2N1c1ccc([N+](=O)[O-])cc1</smiles>

3g; $77 \%$<smiles>CC(C)C1=NC(C(C)C)C(=O)N(c2ccc([N+](=O)[O-])cc2)c2ccccc21</smiles>

3c; $99 \%$ 3h: $70 \%$<smiles>[R]C1=NC([R])C(=O)N(c2ccc([N+](=O)[O-])cc2)c2ccc([X])cc21</smiles><smiles>O=C1CN=C(c2ccccc2)c2ccccc2N1c1ccc([N+](=O)[O-])cc1</smiles>

3d; $82 \%$<smiles>O=C1C(Cc2ccccc2)N=C(c2ccccc2)c2ccccc2N1c1ccc([N+](=O)[O-])cc1</smiles>

3e; $78 \%$ Bradykinin antagonist<smiles>O=C1C(Cc2ccccc2)N=C(c2ccccc2-c2ccccc2F)c2ccccc2N1c1ccc([N+](=O)[O-])cc1</smiles>

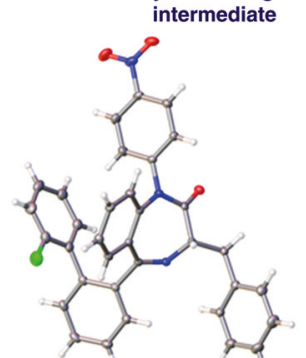

3h

Scheme 1 N-Arylated 1,4-Benzodiazepines 
The use of other unsymmetrical diaryliodonium triflates was also explored (Table 3), which required longer reaction time and led to both aryl groups being transferred to obtain 3i-1. As expected, the transfer of more sterically hindered or less electron-rich groups was preferred. Further attempts to use unsymmetrical diaryliodonium salts such as phenyl(3-methylphenyl)iodonium triflate, phenyl(4-methylphenyl)iodonium triflate, and (2-methylphenyl)(2,4,6trimethylphenyl)iodonium triflate gave little or no products. Additionally, attempted $\mathrm{N}$-arylation with symmetrical diaryliodonium triflates or tetrafluoroborates such as bis(2fluorophenyl)iodonium tetrafluoroborate and bis(4-bromophenyl)iodonium triftlate gave, at best, traces of products.

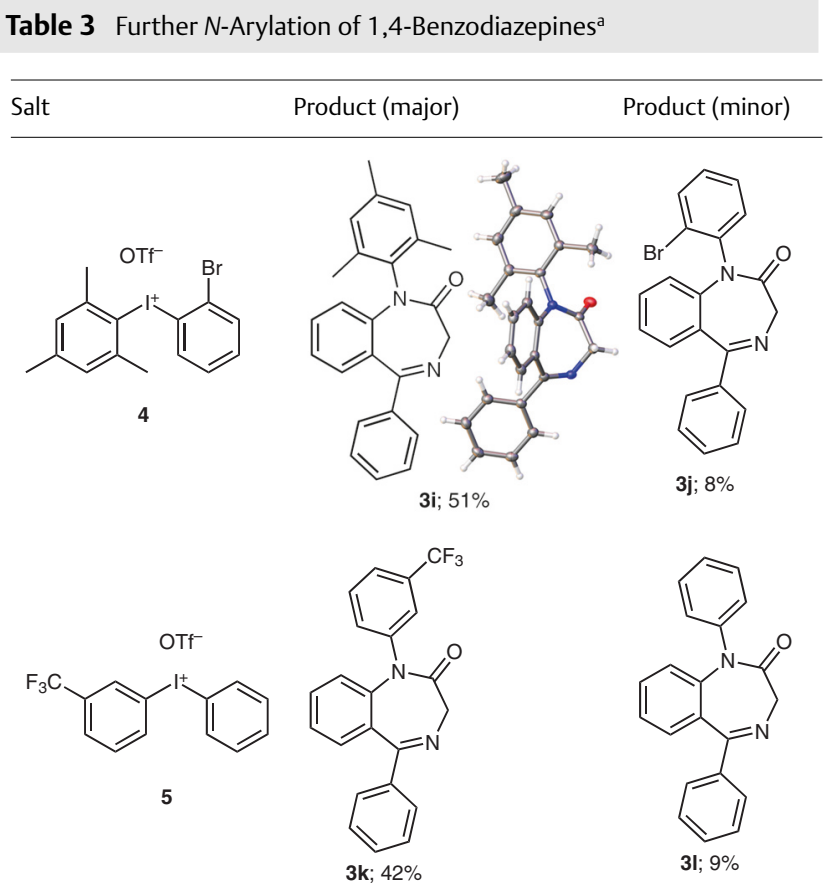

a Reaction time $=8 \mathrm{~h}$

We have briefly explored the $N$-arylation on a $1,3,4-$ benzotriazepine $\mathbf{6}$, which resulted in diarylation and yielded 7 (Scheme 2).<smiles>O=C1NN=C(c2ccccc2)c2ccccc2N1</smiles>

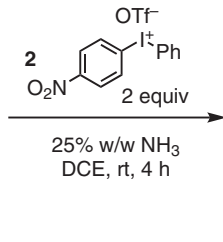

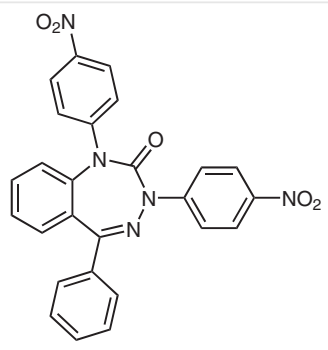

$7 ; 51 \%$
Scheme 2 N-Arylation on a 1,3,4-Benzotriazepine
Interestingly, the iodonium salts were observed to undergo reaction with water present in the reaction to give diarylether products. The ether product is only observed in substantial amounts when the benzodiazepine substrates react poorly with the diaryliodonium salts (Table 4 ). The ether product $\mathbf{1 0}$ was also obtained merely by stirring the iodonium salt with water in DCE with a mild base for 20 min at room temperature with a yield of $43 \%$. Olofsson et al. have reported the synthesis of related diarylethers by reacting diaryliodonium salts with phenols in the presence of mild bases. ${ }^{39}$

Table 4 Diaryl Ether Formation

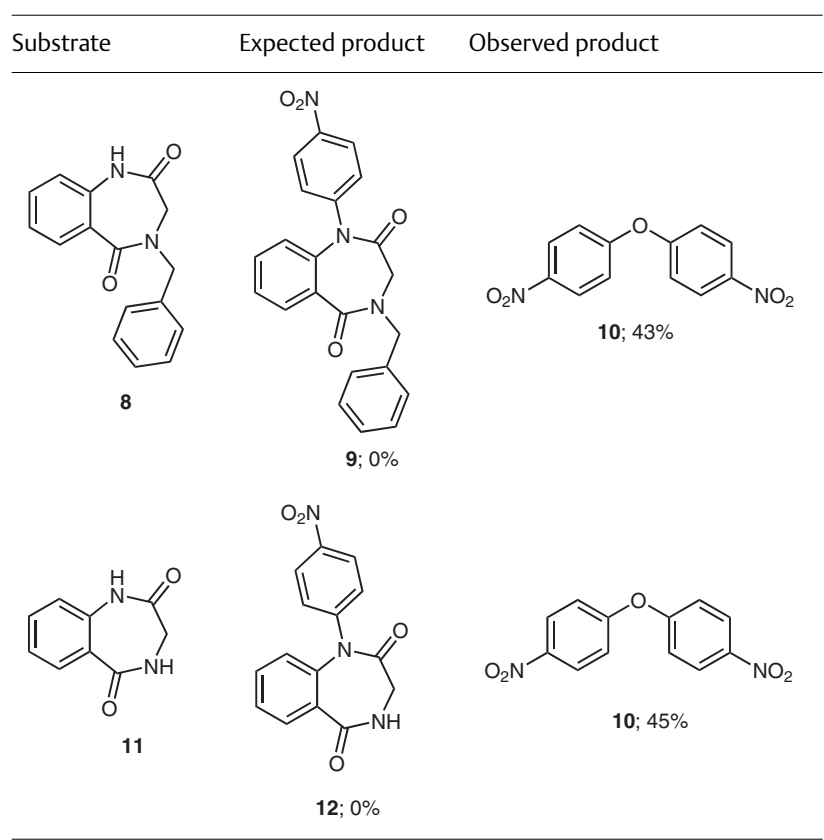

In summary we have presented a mild metal-free route to $\mathrm{N}$-arylated benzodiazepines, three of which were structurally characterized in the solid state ( $\mathbf{3 a}, \mathbf{3 h}, \mathbf{3 i}){ }^{40,41}$

\section{Funding Information}

R.K. is funded by an EPSRC/AZ funded PhD studentship (EP/M507568/1) with additional support from AstraZeneca [14550001 (SME)] and Tocris Biosciences. The EPSRC is also thanked for funding the UK National Crystallography Service

\section{Acknowledgment}

We thank Dr. Alaa Abdul-Sada (Sussex) and the EPSRC UK National Mass Spectrometry Facility at Swansea University for HRMS measurements. 


\section{Supporting Information}

Supporting information for this article is available online at https://doi.org/10.1055/s-0036-1590920.

\section{References and Notes}

(1) Filippakopoulos, P.; Qi, J.; Picaud, S.; Shen, Y.; Smith, W. B.; Fedorov, O.; Morse, E. M.; Keates, T.; Hickman, T. T.; Felletar, I.; Philpott, M.; Munro, S.; McKeown, M. R.; Wang, Y.; Christie, A. L.; West, N.; Cameron, M. J.; Schwartz, B.; Heightman, T. D.; La, Thangue. N.; French, C. A.; Wiest, O.; Kung, A. L.; Knapp, S.; Bradner, J. E. Nature 2010, 468, 1067.

(2) Field, G. F.; Zally, W. J.; Sternbach, L. H. J. Am. Chem. Soc. 1967, $80,332$.

(3) Ellmann, J. A. Acc. Chem. Res. 1996, 29, 132.

(4) Nadin, A.; Sánchez, López. J. M.; Owens, A. P.; Howells, D. M.; Talbot, A. C.; Harrison, T. J. Org. Chem. 2003, 68, 2844.

(5) Baud, M. G.; Lin-Shiao, E.; Cardote, T.; Tallant, C.; Pschibul, A.; Chan, K. H.; Zengerle, M.; Garcia, J. R.; Kwan, T. T.; Ferguson, F. M.; Ciulli, A. Science 2014, 346, 638.

(6) Baud, M. G.; Lin-Shiao, E.; Zengerle, M.; Tallant, C.; Ciulli, A. J. Med. Chem. 2016, 59, 1492.

(7) Mirguet, O.; Gosmini, R.; Toum, J.; Clement, C. A.; Barnathan, M.; Brusq, J. M.; Mordaunt, J. E.; Grimes, R. M.; Crowe, M.; Pineau, O.; Ajakane, M.; Daugan, A.; Jeffrey, P.; Cutler, L.; Haynes, A. C.; Smithers, N. N.; Chung, C. W.; Bamborough, P.; Uings, I. J.; Lewis, A.; Witherington, J.; Parr, N.; Prinjha, R. K.; Nicodeme, E. J. Med. Chem. 2013, 56, 7501.

(8) Liu, J. J.; Higgins, B.; Ju, G.; Kolinsky, K.; Luk, K. C.; Packman, K.; Pizzolato, G.; Ren, Y.; Thakkar, K.; Tovar, C.; Zhang, Z.; Wovkulich, P. M. ACS Med. Chem. Lett. 2013, 4, 259.

(9) Evans, B.; Rittle, K.; Bock, M.; DiPardo, R.; Freidinger, R.; Whitter, W.; Lundell, G.; Veber, D.; Anderson, P.; Chang, R.; Lotti, V.; Cerino, D.; Chen, T.; Kling, P.; Kunkel, K.; Springer, J.; Hirshfield, J. J. Med. Chem. 1988, 31, 2235.

(10) Filippakopoulos, P.; Picaud, S.; Fedorov, O.; Keller, M.; Wrobel, M.; Morgenstern, O.; Bracher, F.; Knapp, S. Bioorg. Med. Chem. 2012, 20, 1878 .

(11) Smith, S. G.; Sanchez, R.; Zhou, M.-M. Chem. Biol. 2014, 573.

(12) Filippakopoulos, P.; Knapp, S. Nat. Rev. Drug Discovery 2014, 13 , 337.

(13) Carter, M. C.; Alber, D. G.; Baxter, R. C.; Bithell, S. K.; Budworth, J.; Chubb, A.; Cockerill, G. S.; Dowdell, V. C. L.; Henderson, E. A.; Keegan, S. J.; Kelsey, R. D.; Lockyer, M. J.; Stables, J. N.; Wilson, L. J.; Powell, K. L. J. Med. Chem. 2006, 49, 2311.

(14) Ghelani, S. M.; Naliapara, Y. T. J. Heterocycl. Chem. 2016, 53, 1795.

(15) Abdelkafi, H.; Cintrat, J. C. Sci. Rep. 2015, 5, 12131.

(16) Kaur, N. Int. J. Pharm. Biol. Sci. 2013, 485.

(17) Hu, X.; Dong, Y.; Liu, G. Mol. Diversity 2015, 19, 695.

(18) Spencer, J.; Rathnam, R.; Chowdhry, B. Future Med. Chem. 2010, $2,1441$.

(19) Dzladulewlcz, E. K.; Brown, M. C.; Dunstan, A. R.; Lee, W.; Said, N. B.; Garratt, P. J. Bioorg. Med. Chem. Lett. 1999, 9, 463.

(20) McDonald, I.; Austin, C.; Buck, I.; Dunstone, D.; Gaffen, J.; Griffin, E.; Harper, E.; Hull, R.; Kalindjian, S.; Linney, I.; Low, C.; Patel, D.; Pether, M.; Raynor, M.; Roberts, S.; Shaxted, M.; Spencer, J.; Steel, K.; Sykes, D.; Wright, P.; Xun, W. J. Med. Chem. 2007, 50, 4789.

(21) Cuny, G.; Bois-Choussy, M. L.; Zhu, J.J. Am. Chem. Soc. 2004, 126, 14475.
(22) Klapars, A.; Antilla, J. C.; Huang, X.; Buchwald, S. L. J. Am. Chem. Soc. 2001, 123, 7727

(23) Talukdar, D.; Das, G.; Thakur, S.; Karak, N.; Thakur, A. J. Catal. Commun. 2015, 59, 238.

(24) Strittmatter, S. M. Nat. Med. 2014, 20, 590.

(25) Corsello, S. M.; Bittker, J. A.; Liu, Z.; Gould, J.; McCarren, P.; Hirschman, J. E.; Johnston, S. E.; Vrcic, A.; Wong, B.; Khan, M.; Asiedu, J.; Narayan, R.; Mader, C. C.; Subramanian, A.; Golub, T. R. Nat. Med. 2017, 23, 405.

(26) Spencer, J.; Rathnam, R. P.; Harvey, A. L.; Clements, C. J.; Clark, R. L.; Barrett, M. P.; Wong, P. E.; Male, L.; Coles, S. J.; Mackay, S. P. Bioorg. Med. Chem. 2011, 19, 1802.

(27) Clark, R. L.; Clements, C. J.; Barrett, M. P.; Mackay, S. P.; Rathnam, R. P.; Owusu-Dapaah, G.; Spencer, J.; Huggan, J. K. Bioorg. Med. Chem. 2012, 20, 6019.

(28) Spencer, J.; Chowdhry, B. Z.; Mallet, A. I.; Rathnam, R. P.; Adatia, T.; Bashall, A.; Rominger, F. Tetrahedron 2008, 64, 6082.

(29) Khan, R.; Felix, R.; Kemmitt, P. D.; Coles, S. J.; Day, I. J.; Tizzard, G. J.; Spencer, J. Adv. Synth. Catal. 2016, 358, 98.

(30) Merritt, E. A.; Olofsson, B. Angew. Chem. Int. Ed. 2009, 48, 9052.

(31) Bielawski, M.; Aili, D.; Olofsson, B. J. Org. Chem. 2008, 73, 4602.

(32) (a) Yusubov, M. S.; Maskaev, A. V.; Zhdankin, V. V. ARKIVOC 2011, 370.

(33) Ghosh, R.; Olofsson, B. Org. Lett. 2014, 16, 1830.

(34) Tinnis, F.; Stridfeldt, E.; Lundberg, H.; Adolfsson, H.; Olofsson, B. Org. Lett. 2015, 17, 2688.

(35) Malmgren, J.; Santoro, S.; Jalalian, N.; Himo, F.; Olofsson, B. Chem. Eur. J. 2013, 19, 10334.

(36) Gonda, Z.; Novak, Z. Chem. Eur. J. 2015, 21, 16801.

(37) Lanning, M.; Fletcher, S. Future Med. Chem. 2013, 5, 2157.

(38) Azzarito, V.; Long, K.; Murphy, N. S.; Wilson, A. J. Nat. Chem. 2013, 5, 161.

(39) Jalalian, N.; Ishikawa, E. E.; Silva, L. F. Jr.; Olofsson, B. Org. Lett. 2011, 13, 1552 .

(40) Coles, S. J.; Gale, P. A. Chem. Sci. 2012, 3, 683.

(41) All reactions were conducted under an inert atmosphere unless specified otherwise. All commercially purchased materials and solvents were used without further purification unless specified otherwise. NMR spectra were recorded on a Varian VNMRS 500 $\left({ }^{1} \mathrm{H}: 500 \mathrm{MHz},{ }^{13} \mathrm{C}: 126 \mathrm{MHz}\right)$ spectrometer and prepared in deuterated solvents such as $\mathrm{CDCl}_{3}$ and DMSO- $d_{6} \cdot{ }^{1} \mathrm{H}$ and ${ }^{13} \mathrm{C}$ chemical shifts were recorded in parts per million (ppm). Multiplicity of ${ }^{1} \mathrm{H}$ NMR peaks are indicated by $\mathrm{s}$ - singlet, $\mathrm{d}$ - doublet, $\mathrm{dd}$ - doublets of doublets, $\mathrm{t}$ - triplet, pt - pseudo triplet, q - quartet, $\mathrm{m}$ - multiplet, and coupling constants are given in Hertz (Hz). Electrospray ionisation-high resolution mass spectra (ESI-HRMS) were obtained using a Bruker Daltonics Apex III where Apollo ESI was used as the ESI source. The molecular ion peaks $[\mathrm{M}]^{+}$were recorded as mass to charge $\mathrm{m} / \mathrm{z}$ ratio.

(42) LC-MS spectra were acquired using a Shimadzu LC-MS 2020, on a Gemini $5 \mu \mathrm{m}$ C18 $110 \AA ̊$ column and percentage purities were run over $30 \mathrm{~min}$ in water/acetonitrile with $0.1 \%$ formic acid $(5$ min at $5 \%, 5-95 \%$ over $20 \mathrm{~min}, 5 \mathrm{~min}$ at $95 \%$ ) with the UV detector at $254 \mathrm{~nm}$. Purifications were performed by flash chromatography on silica gel columns or C18 columns using a Combi flash RF 75 PSI, ISCO unit.

\section{General Procedure}

To a stirred solution of the appropriate 1,4-benzodiazepine or 1,3,4-benzotriazepine $(0.030-1.00 \mathrm{mmol}, 1$ equiv) and diaryliodonium salt $(0.033-1.10 \mathrm{mmol}, 1.1$ equiv) in DCE (5-10 $\mathrm{mL}$ ) was added $25 \% \mathrm{w} / \mathrm{w} \mathrm{NH}_{3}$ solution (aq. $5-10 \mathrm{~mL}$ ), and the reaction mixture was stirred for 30 min (unless stated other- 
wise). Upon completion, the reaction mixture was diluted with dichloromethane $(3 \times 15 \mathrm{~mL})$, and the layers were separated. Combined organic layers were dried $\left(\mathrm{MgSO}_{4}\right)$, concentrated under reduced pressure, and purified by column chromatography, hexane/ethyl acetate (80:20 to 30:70).

1-(4-Nitrophenyl)-5-methyl-1,3-dihydro-2H-1,4-benzodiazepin-2-one (3a)

The product was obtained as white solid $(0.60 \mathrm{mmol}$ scale, 170 $\mathrm{mg}, 96 \%$ ). ${ }^{1} \mathrm{H}$ NMR (500 MHz, $\mathrm{CDCl}_{3}$ ): $\delta=8.27-8.21$ (m, ArH, 2 $\mathrm{H}), 7.62\left(\mathrm{dd}, 3_{\mathrm{HH}}=7.5,1.5 \mathrm{~Hz}, \mathrm{ArH}, 1 \mathrm{H}\right), 7.40-7.35(\mathrm{~m}, \mathrm{ArH}, 3$ H), 7.34-7.29 (m, ArH, $1 \mathrm{H}), 6.82\left(\mathrm{~d},{ }^{3} J_{\mathrm{HH}}=8.0 \mathrm{~Hz}, \mathrm{ArH}, 1 \mathrm{H}\right), 4.70$ $\left(\mathrm{d},{ }^{2} J_{\mathrm{HH}}=10.5 \mathrm{~Hz}, \mathrm{COCH}_{2}, 1 \mathrm{H}\right), 3.83\left(\mathrm{~d},{ }^{2} J_{\mathrm{HH}}=10.5 \mathrm{~Hz}, \mathrm{COCH}_{2}, 1\right.$ $\mathrm{H}), 2.62\left(\mathrm{~s}, \mathrm{CH}_{3}, 3 \mathrm{H}\right) .{ }^{13} \mathrm{C}$ NMR $\left(126 \mathrm{MHz}, \mathrm{CDCl}_{3}\right): \delta=170.1$ $(\mathrm{C}=\mathrm{O}), 168.1(\mathrm{C}=\mathrm{N}), 146.7(\operatorname{ArC}), 146.0(\operatorname{ArC}), 140.8(\operatorname{ArC}), 131.4$ (ArC), $131.3(\operatorname{ArC}), 128.7(\operatorname{ArC} \times 2), 127.8(\operatorname{ArC}), 125.9(\operatorname{ArC})$, $125.1(\mathrm{ArC}), 124.5(\mathrm{ArC} \times 2), 56.6\left(\mathrm{COCH}_{2}\right), 25.5\left(\mathrm{CH}_{3}\right)$. ESIHRMS: $m / z$ calcd for $\mathrm{C}_{16} \mathrm{H}_{13} \mathrm{~N}_{3} \mathrm{O}_{3}[+\mathrm{H}]^{+}:$296.1030; found: 296.1033. LC-MS purity (UV) $=100 \%, t_{R}=8.10 \mathrm{~min}$.

1-(4-Nitrophenyl)-5-(propan-2-yl)-1,3-dihydro-2H-1,4-benzodiazepin-2-one (3b)

The product was obtained as a white solid $(0.52 \mathrm{mmol}$ scale, $166 \mathrm{mg}, 99 \%$ ). ${ }^{1} \mathrm{H}$ NMR (500 MHz, $\mathrm{CDCl}_{3}$ ): $\delta=8.27-8.20$ (m, ArH, $2 \mathrm{H}$ ), 7.59 (dd, $\left.{ }^{3} J_{\mathrm{HH}}=7.5,2.0 \mathrm{~Hz}, \mathrm{ArH}, 1 \mathrm{H}\right), 7.39-7.35$ (m, ArH, 3 $\mathrm{H}), 7.34-7.30(\mathrm{~m}, \mathrm{ArH}, 1 \mathrm{H}), 6.83\left(\mathrm{dd},{ }^{3} J_{\mathrm{HH}}=8.0,1.5 \mathrm{~Hz}, \mathrm{ArH}, 1\right.$ $\mathrm{H}), 4.72\left(\mathrm{~d},{ }^{2} J_{\mathrm{HH}}=10.5 \mathrm{~Hz}, \mathrm{COCH}_{2}, 1 \mathrm{H}\right), 3.82\left(\mathrm{~d},{ }^{2} J_{\mathrm{HH}}=10.5 \mathrm{~Hz}\right.$, $\left.\mathrm{COCH}_{2}, 1 \mathrm{H}\right), 3.34-3.25(\mathrm{~m}, 1 \mathrm{H}), 1.35\left(\mathrm{~d},{ }^{3} J_{\mathrm{HH}}=7.0 \mathrm{~Hz}\right.$, $\left.\mathrm{CNCHC}_{2} \mathrm{CH}_{6}, 3 \mathrm{H}\right), 1.11\left(\mathrm{~d},{ }^{3} J_{\mathrm{HH}}=7.0 \mathrm{~Hz}, \mathrm{CNCHC}_{2} \mathrm{CH}_{6}, 3 \mathrm{H}\right) .{ }^{13} \mathrm{C}$ NMR $\left(126 \mathrm{MHz}, \mathrm{CDCl}_{3}\right): \delta=176.9(\mathrm{C}=\mathrm{O}), 168.7(\mathrm{C}=\mathrm{N}), 146.7$ (ArC), $145.9(\operatorname{ArC}), 141.5(\operatorname{ArC}), 131.6(\operatorname{ArC}), 130.9(\operatorname{ArC}), 128.3$ $(\operatorname{ArC} \times 2), 127.0(\operatorname{ArC}), 126.0(\operatorname{ArC}), 125.0(\operatorname{ArC}), 124.5(\operatorname{ArC} \times 2)$, $56.5\left(\mathrm{COCH}_{2}\right), \quad 35.6\left(\mathrm{CNCHC}_{2} \mathrm{H}_{6}\right), 22.0\left(\mathrm{CNCHC}_{2} \mathrm{H}_{6}\right), \quad 19.2$ $\left(\mathrm{CNCHC}_{2} \mathrm{H}_{6}\right)$. ESI-HRMS: $\mathrm{m} / z$ calcd for $\mathrm{C}_{18} \mathrm{H}_{17} \mathrm{~N}_{3} \mathrm{O}_{3} \quad[+\mathrm{H}]^{+}$: 324.1270; found: 324.1281 . LC-MS purity (UV) $=96 \%, t_{\mathrm{R}}=$ $18.73 \mathrm{~min}$.

1-(4-Nitrophenyl)-3-(propan-2-yl)-5-(propan-2-yl)-1,3dihydro-2H-1,4-benzodiazepin-2-one (3c)

The product was obtained as white solid $(0.25 \mathrm{mmol}$ scale, 91 $\mathrm{mg}, 99 \%) .{ }^{1} \mathrm{H}$ NMR $(500 \mathrm{MHz}) \mathrm{CDCl}_{3}: \delta=8.25-8.18(\mathrm{~m}, \mathrm{ArH}, 2$ $\mathrm{H}), 7.61\left(\mathrm{dd},{ }^{3} \mathrm{H}_{\mathrm{HH}}=8.0,1.5 \mathrm{~Hz}, \mathrm{ArH}, 1 \mathrm{H}\right), 7.39-7.24(\mathrm{~m}, \mathrm{ArH}, 4 \mathrm{H})$, $6.85(\mathrm{dd}, J=8.0,1.5 \mathrm{~Hz}, \mathrm{ArH}, 1 \mathrm{H}), 3.27$ (hept, ${ }^{3} J_{\mathrm{HH}}=7.0 \mathrm{~Hz}$, $\left.\mathrm{CNCHCH}_{3} \mathrm{CH}_{3}, 1 \mathrm{H}\right), 3.12\left(\mathrm{~d},{ }^{3} \mathrm{~J}_{\mathrm{HH}}=9.5 \mathrm{~Hz}, \mathrm{COCHCHC} \mathrm{H}_{6}, 1 \mathrm{H}\right)$, 2.72-2.61 (m, COCHCHC $\left.{ }_{2} \mathrm{H}_{6}, 1 \mathrm{H}\right), 1.33\left(\mathrm{~d},{ }^{3} \mathrm{JHH}_{\mathrm{HH}}=7.0 \mathrm{~Hz}\right.$, $\left.\mathrm{CNCHC}_{2} \mathrm{H}_{6}, 3 \mathrm{H}\right), 1.07\left(\mathrm{~d},{ }^{3} J_{\mathrm{HH}}=7.0 \mathrm{~Hz}, \mathrm{CNCHC}_{2} \mathrm{CH}_{6}, 3 \mathrm{H}\right), 1.05-$ $1.02\left(\mathrm{~m}, \mathrm{COCHCHC}{ }_{2} \mathrm{H}_{6}, 6 \mathrm{H}\right) .{ }^{13} \mathrm{C}$ NMR $\left(126 \mathrm{MHz}, \mathrm{CDCl}_{3}\right): \delta=$ $173.9(\mathrm{C}=\mathrm{O}), 168.3(\mathrm{C}=\mathrm{N}), 147.4(\operatorname{ArC}), 145.7(\operatorname{ArC}), 141.1(\operatorname{ArC})$, $131.9(\mathrm{ArC}), 130.6(\mathrm{ArC}), 128.4(\operatorname{ArC} \times 2), 126.8(\mathrm{ArC}), 125.7$ ( $\mathrm{ArC}), 125.1(\mathrm{ArC}), 124.4(\mathrm{ArC} \times 2), 69.3\left(\mathrm{COCHCHC}_{2} \mathrm{H}_{6}\right), 35.5$ $\left(\mathrm{CNCHCH}_{3} \mathrm{CH}_{3}\right), 22.2\left(\mathrm{COCHCHC}_{2} \mathrm{H}_{6}\right), 21.9\left(\mathrm{CNCHC}_{2} \mathrm{H}_{6}\right), 20.1$, $\left(\mathrm{CNCHC}_{2} \mathrm{H}_{6}\right) 19.3\left(\mathrm{COCHCHC}_{2} \mathrm{H}_{6}\right), 18.7\left(\mathrm{COCHCHC}_{2} \mathrm{H}_{6}\right)$. ESIHRMS: $\mathrm{m} / \mathrm{z}$ calcd for $\mathrm{C}_{21} \mathrm{H}_{23} \mathrm{~N}_{3} \mathrm{O}_{3}[+\mathrm{H}]^{+}:$366.1812; found: 366.1816. LC-MS purity (UV) $=95 \%, t_{\mathrm{R}}=23.47 \mathrm{~min}$.

\section{1-(4-Nitrophenyl)-5-phenyl-1,3-dihydro-2H-1,4-benzodiaz-} epin-2-one (3d)

The product was obtained as white solid $(0.60 \mathrm{mmol}$ scale, 176 $\mathrm{mg}, 82 \%) .{ }^{1} \mathrm{H}$ NMR $\left(500 \mathrm{MHz}, \mathrm{CDCl}_{3}\right): \delta=8.30-8.23(\mathrm{~m}, \mathrm{ArH}, 2$ H), 7.77-7.71 (m, ArH, 2 H), 7.55-7.51 (m, ArH, $1 \mathrm{H}), 7.49-7.45$ $(\mathrm{m}, \mathrm{ArH}, 3 \mathrm{H}), 7.45-7.41(\mathrm{~m}, \mathrm{ArH}, 3 \mathrm{H}), 7.29\left(\mathrm{~d},{ }^{3} J_{\mathrm{HH}}=8.0 \mathrm{~Hz}\right.$, $\mathrm{ArH}, 1 \mathrm{H}), 6.94\left(\mathrm{~d},{ }^{3} J_{\mathrm{HH}}=8.0 \mathrm{~Hz}, \mathrm{ArH}, 1 \mathrm{H}\right), 4.96\left(\mathrm{~d},{ }^{2} J_{\mathrm{HH}}=10.5 \mathrm{~Hz}\right.$, $\left.\mathrm{COCH}_{2}, 1 \mathrm{H}\right), 4.03\left(\mathrm{~d},{ }^{2} J_{\mathrm{HH}}=10.5 \mathrm{~Hz}, \mathrm{COCH}_{2}, 1 \mathrm{H}\right) .{ }^{13} \mathrm{C}$ NMR $(126$ $\left.\mathrm{MHz}, \mathrm{CDCl}_{3}\right): \delta=170.3(\mathrm{C}=\mathrm{O}), 168.3(\mathrm{C}=\mathrm{N}), 146.7(\mathrm{ArC}), 146.0$ (ArC), 142.7 (ArC), $138.4(\operatorname{ArC}), 131.4(\operatorname{ArC}), 130.8(\operatorname{ArC}), 130.4$ (ArC), $130.3(\operatorname{ArC}), 129.4(\operatorname{ArC} \times 2), 128.5(\operatorname{ArC} \times 2), 128.4(\operatorname{ArC} \times$ 2), $125.4(\mathrm{ArC}), 125.0(\mathrm{ArC}), 124.5(\mathrm{ArC} \times 2), 57.4\left(\mathrm{COCH}_{2}\right)$. ESI-
HRMS: $m / z$ calcd for $\mathrm{C}_{21} \mathrm{H}_{15} \mathrm{~N}_{3} \mathrm{O}_{3}[+\mathrm{H}]^{+}$: 358.1186; found: 358.1187. LC-MS purity (UV) $=95 \%, t_{\mathrm{R}}=18.35 \mathrm{~min}$.

1-(4-Nitrophenyl)-3-benzyl-5-phenyl-1,3-dihydro-2H-1,4benzodiazepin-2-one (3e)

The product was obtained as white solid $(0.40 \mathrm{mmol}$ scale, 140 $\mathrm{mg}, 78 \%$ ). ${ }^{1} \mathrm{H}$ NMR $\left(500 \mathrm{MHz}, \mathrm{CDCl}_{3}\right): \delta=8.27-8.21$ (m, ArH, 2 $\mathrm{H}), 7.67\left(\mathrm{~d},{ }^{3} \mathrm{~J}_{\mathrm{HH}}=7.5 \mathrm{~Hz}, \mathrm{ArH}, 2 \mathrm{H}\right), 7.52-7.48(\mathrm{~m}, 1 \mathrm{H}), 7.47-$ 7.43 (m, ArH, 2 H), 7.41-7.37 (m, ArH, 5 H), 7.36-7.30 (m, ArH, 3 H), 7.25-7.21 (m, ArH, $2 \mathrm{H}), 6.90\left(\mathrm{~d},{ }^{3} \mathrm{~J}_{\mathrm{HH}}=8.0 \mathrm{~Hz}, \mathrm{ArH}, 1 \mathrm{H}\right), 4.01$ (dd, $\left.J=7.5,6.0 \mathrm{~Hz}, \mathrm{COCHCH}_{2}, 1 \mathrm{H}\right), 3.68\left(\mathrm{dd},{ }^{2,3} \mathrm{JHH}_{\mathrm{HH}}=14.0,6.0 \mathrm{~Hz}\right.$, $\left.\mathrm{COCHCH}_{2}, 1 \mathrm{H}\right), 3.62\left(\mathrm{dd},{ }^{2,3} J_{\mathrm{HH}}=14.0,7.5 \mathrm{~Hz}, \mathrm{COCHCH}_{2}, 1 \mathrm{H}\right) .{ }^{13} \mathrm{C}$ NMR (126 MHz, $\left.\mathrm{CDCl}_{3}\right): \delta=168.8(\mathrm{C}=\mathrm{O}), 168.5(\mathrm{C}=\mathrm{N}), 147.1$ (ArC), 145.9 (ArC), 142.1 (ArC), $138.9(\operatorname{ArC}), 138.4(\operatorname{ArC}), 131.5$ (ArC), $130.8(\operatorname{ArC}), 130.5(\operatorname{ArC}), 130.3(\operatorname{ArC}), 130.0(\operatorname{ArC} \times 2)$, $129.5(\operatorname{ArC} \times 2), 128.6(\operatorname{ArC} \times 2), 128.5(\operatorname{ArC} \times 2), 128.3(\operatorname{ArC} \times 2)$, 126.3 (ArC), $125.3(\operatorname{ArC}), 125.1(\operatorname{ArC}), 124.5(\operatorname{ArC} \times 2), 65.6$ $\left(\mathrm{COCHCH}_{2}\right), 37.9\left(\mathrm{COCHCH}_{2}\right)$. ESI-HRMS: $\mathrm{m} / z$ calcd for $\mathrm{C}_{28} \mathrm{H}_{21} \mathrm{~N}_{3} \mathrm{O}_{3}[+\mathrm{H}]^{+}:$448.1656; found: 448.1669. LC-MS purity $(\mathrm{UV})=99 \%, t_{\mathrm{R}}=20.81 \mathrm{~min}$.

7-Chloro-1-(4-nitrophenyl)-3-benzyl-5-phenyl-1,3-dihydro2H-1,4-benzodiazepin-2-one (3f)

The product was obtained as white solid $(0.15 \mathrm{mmol}$ scale, 54 mg, 75\%). ${ }^{1} \mathrm{H}$ NMR $\left(500 \mathrm{MHz}, \mathrm{CDCl}_{3}\right): \delta=8.26\left(\mathrm{~d},{ }^{3} J_{\mathrm{HH}}=8.5 \mathrm{~Hz}\right.$, ArH, $2 \mathrm{H}), 7.66\left(\mathrm{~d},{ }^{3} J_{\mathrm{HH}}=7.5 \mathrm{~Hz}, \mathrm{ArH}, 2 \mathrm{H}\right), 7.57-7.50(\mathrm{~m}, \mathrm{ArH}, 1$ H), 7.51-7.44 (m, ArH, 2 H), 7.42-7.36 (m, ArH, $3 \mathrm{H}), 7.34-7.30$ $(\mathrm{m}, \operatorname{ArH}, 2 \mathrm{H}), 7.26(\mathrm{~s}, \mathrm{ArH}, 3 \mathrm{H}), 7.17(\mathrm{~d}, J=8.7 \mathrm{~Hz}, \operatorname{ArH}, 1 \mathrm{H})$, $6.85\left(\mathrm{~d},{ }^{3} J_{H H}=8.5 \mathrm{~Hz}, \mathrm{ArH}, 1 \mathrm{H}\right), 3.99(\mathrm{dd}, J=7.5,6.0 \mathrm{~Hz}$, $\left.\mathrm{COCHCH}_{2}, 1 \mathrm{H}\right), 3.70-3.57\left(\mathrm{~m}, \mathrm{COCHCH}_{2}, 2 \mathrm{H}\right) .{ }^{13} \mathrm{C}$ NMR $(126$ $\left.\mathrm{MHz}, \mathrm{CDCl}_{3}\right): \delta=168.4,(\mathrm{C}=\mathrm{O}), 167.2(\mathrm{C}=\mathrm{N}), 146.6(\mathrm{ArC}), 146.1$ (ArC), 140.6 (ArC), 138.6 (ArC), 137.7 (ArC), 131.7 (ArC), 131.1 $(\operatorname{ArC}), 129.9(\operatorname{ArC} \times 2), 129.8(\operatorname{ArC}), 129.5(\operatorname{ArC} \times 2), 128.7(\operatorname{ArC} \times$ 2), $128.6(\operatorname{ArC} \times 2), 128.3(\operatorname{ArC} \times 2), 126.5(\operatorname{ArC}), 126.4(\operatorname{ArC})$, 126.2 (ArC), $124.6(\mathrm{ArC} \times 2), 119.3(\mathrm{ArC}) 65.8\left(\mathrm{COCHCH}_{2}\right), 37.9$ $\left(\mathrm{COCHCH}_{2}\right)$. ESI-HRMS: $\mathrm{m} / \mathrm{z}$ calcd for $\mathrm{C}_{28} \mathrm{H}_{20} \mathrm{ClN}_{3} \mathrm{O}_{3}[+\mathrm{H}]^{+}$: 482.1266; found: 482.1286 . LC-MS purity $(\mathrm{UV})=95 \%, t_{\mathrm{R}}=19.71$ min.

1-(4-Nitrophenyl)-3-benzyl-5-(pyridine-2-yl)-1,3-dihydro2H-1,4-benzodiazepin-2-one (3g)

The product was obtained as white solid $(0.11 \mathrm{mmol}$ scale, 38 $\mathrm{mg}, 77 \%) .{ }^{1} \mathrm{H}$ NMR $\left(500 \mathrm{MHz}, \mathrm{CDCl}_{3}\right.$ ): $\delta=8.67-8.62(\mathrm{~m}, \mathrm{ArH}, 1$ $\mathrm{H}), 8.15\left(\mathrm{~d},{ }^{3} \mathrm{JHH}_{\mathrm{HH}}=8.0 \mathrm{~Hz}, \mathrm{ArH}, 2 \mathrm{H}\right), 8.18-8.12(\mathrm{~m}, \mathrm{ArH}, 1 \mathrm{H})$, 7.88-7.81 (m, ArH, $1 \mathrm{H}), 7.44-7.42$ (m, ArH, $2 \mathrm{H}), 7.42-7.37$ (m, ArH, 4 H), 7.35-7.26 (m, ArH, 3 H), 7.25-7.21 (m, ArH, 2 H), 6.89 $\left(\mathrm{d},{ }^{3} \mathrm{HH}_{\mathrm{HH}}=8.0 \mathrm{~Hz}, \mathrm{ArH}, 1 \mathrm{H}\right), 4.10\left(\mathrm{dd},{ }^{3} J_{\mathrm{HH}}=8.0,6.0 \mathrm{~Hz}, \mathrm{COCHCH}_{2}\right.$, $1 \mathrm{H}), 3.70\left(\mathrm{dd}^{2,3} J_{\mathrm{HH}}=14.0,7.0 \mathrm{~Hz}, \mathrm{COCHCH}_{2}, 1 \mathrm{H}\right), 3.62\left(\mathrm{dd},{ }^{2,3} J_{\mathrm{HH}}\right.$ $\left.=14.0,7.5 \mathrm{~Hz}, \mathrm{COCHCH}_{2}, 1 \mathrm{H}\right) .{ }^{13} \mathrm{C}$ NMR $\left(126 \mathrm{MHz}, \mathrm{CDCl}_{3}\right): \delta=$ 168.6 $(\mathrm{C}=\mathrm{O}), 167.6(\mathrm{C}=\mathrm{N}), 155.9(\operatorname{ArC}), 148.7(\operatorname{ArC}), 147.1(\operatorname{ArC})$, 145.9 (ArC), 141.9 (ArC), 138.9 (ArC), 136.8 (ArC), $131.4(\operatorname{ArC})$, $130.8(\operatorname{ArC}), 129.9(\operatorname{ArC} \times 2), 128.8(\operatorname{ArC} \times 2), 128.3(\operatorname{ArC} \times 2)$, $126.3(\mathrm{ArC}), 125.2(\mathrm{ArC}), 125.1(\mathrm{ArC}), 124.8(\mathrm{ArC} \times 2), 124.4(\mathrm{ArC}$ $\times 2), 123.8(\mathrm{ArC}), 65.8\left(\mathrm{COCHCH}_{2}\right), 37.8\left(\mathrm{COCHCH}_{2}\right)$. ESI-HRMS: $m / z$ calcd for $\mathrm{C}_{27} \mathrm{H}_{20} \mathrm{~N}_{4} \mathrm{O}_{3}[+\mathrm{H}]^{+}:$449.1608; found: 449.1617. LCMS purity $(\mathrm{UV})=99 \%, t_{\mathrm{R}}=20.81 \mathrm{~min}$.

1-(4-Nitrophenyl)-3-benzyl-5-(2'-fluorobiphenyl-2-yl)-1,3dihydro-2H-1,4-benzodiazepin-2-one ( $3 \mathrm{~h}$ )

The product was obtained as white solid $(0.03 \mathrm{mmol}$ scale, 11 mg, 70\%). ${ }^{1} \mathrm{H}$ NMR $\left(500 \mathrm{MHz}, \mathrm{CDCl}_{3}\right): \delta=8.14\left(\mathrm{~d},{ }^{3} J_{\mathrm{HH}}=8.5 \mathrm{~Hz}\right.$, ArH, 2 H), 7.57-7.52 (m, ArH, 1 H), 7.51-7.45 (m, ArH, 1 H), 7.42 $\left(\mathrm{d},{ }^{3} J_{\mathrm{HH}}=7.5 \mathrm{~Hz}, \mathrm{ArH}, 1 \mathrm{H}\right), 7.38\left(\mathrm{~d},{ }^{3} J_{\mathrm{HH}}=7.5 \mathrm{~Hz}, \mathrm{ArH}, 1 \mathrm{H}\right), 7.32-$ 7.27 (m, ArH, 7 H), 7.26-7.19 (m, ArH, 2 H), 7.15-7.09 (m, ArH, 1 H), $7.06\left(\mathrm{~d},{ }^{3} J_{H H}=7.5 \mathrm{~Hz}, \mathrm{ArH}, 1 \mathrm{H}\right), 7.00-6.93(\mathrm{~m}, \mathrm{ArH}, 3 \mathrm{H}), 6.65$ $\left(\mathrm{d},{ }^{3} J_{\mathrm{HH}}=8.5 \mathrm{~Hz}, \mathrm{ArH}, 1 \mathrm{H}\right), 3.80\left(\mathrm{dd},{ }^{3} J_{\mathrm{HH}}=8.0,5.5 \mathrm{~Hz}, \mathrm{COCHCH}_{2}\right.$, $1 \mathrm{H}), 3.69\left(\mathrm{~d},{ }^{3} J_{\mathrm{HH}}=8.0 \mathrm{~Hz}, \mathrm{COCHCH}_{2}, 1 \mathrm{H}\right), 3.66\left(\mathrm{~d},{ }^{3} J_{\mathrm{HH}}=8.0 \mathrm{~Hz}\right.$, 
$\left.\mathrm{COCHCH}_{2}, 1 \mathrm{H}\right) .{ }^{13} \mathrm{C}$ NMR $\left(126 \mathrm{MHz}, \mathrm{CDCl}_{3}\right): \delta=169.5(\mathrm{C}=0)$, $167.9(\mathrm{C}=\mathrm{N}), 159.2\left(\mathrm{~d},{ }^{1} \mathrm{JFC}_{\mathrm{FC}}=247.5 \mathrm{~Hz}, \operatorname{ArC}\right) 147.1(\mathrm{ArC}), 145.7$ (ArC), 141.5 (ArC), 138.8 (ArC), $138.7(\operatorname{ArC}), 135.7(\operatorname{ArC}), 132.0(\mathrm{~d}$, $\left.{ }^{3} J_{\mathrm{FC}}=3.5 \mathrm{~Hz}, \operatorname{ArC}\right), 131.6(\operatorname{ArC}), 131.4(\operatorname{ArC}), 130.8(\operatorname{ArC}), 130.3$ $(\operatorname{ArC}), 129.9(\operatorname{ArC} \times 2), 129.8(\operatorname{ArC}), 129.5(\operatorname{ArC}), 129.2(\operatorname{ArC})$, $128.9\left(\mathrm{~d},{ }^{3} J_{\mathrm{FC}}=8.0 \mathrm{~Hz}, \operatorname{ArC}\right), 128.5(\operatorname{ArC} \times 2), 128.3(\operatorname{ArC} \times 2)$, $128.1(\mathrm{ArC}), 126.3(\mathrm{ArC}), 125.2(\operatorname{ArC}), 124.8(\mathrm{ArC}), 124.5\left(\mathrm{~d},{ }^{4} \mathrm{JFC}_{\mathrm{FC}}=\right.$ $3.5 \mathrm{~Hz}, \mathrm{ArC}), 124.2(\mathrm{ArC} x \mathrm{2}), 115.45\left(\mathrm{~d},{ }^{2} J_{\mathrm{FC}}=22.0 \mathrm{~Hz}, \mathrm{ArC}\right) 66.0$ $\left(\mathrm{COCHCH}_{2}\right), 37.8\left(\mathrm{COCHCH}_{2}\right)$. ESI-HRMS: $\mathrm{m} / \mathrm{z}$ calcd for $\mathrm{C}_{34} \mathrm{H}_{24} \mathrm{~N}_{3} \mathrm{O}_{3}[+\mathrm{H}]^{+}:$542.1874; found: 542.1881. LC-MS purity $(\mathrm{UV})=93 \%, t_{\mathrm{R}}=23.27 \mathrm{~min}$.

\section{1-(2,4,6-Trimethylphenyl)-5-phenyl-1,3-dihydro-2H-1,4-} benzodiazepin-2-one ( $3 \mathrm{i})$

The reaction was run for $8 \mathrm{~h}$. The product was obtained as white solid (1.00 mmol scale, $181 \mathrm{mg}, 51 \%) .{ }^{1} \mathrm{H}$ NMR (500 MHz, DMSO- $\left.d_{6}\right): \delta=7.60-7.57$ (m, ArH, $2 \mathrm{H}$ ), 7.54-7.46 (m, ArH, $4 \mathrm{H}$ ), $7.32\left(\mathrm{~d},{ }^{3} J_{H H}=8.0 \mathrm{~Hz}, \mathrm{ArH}, 1 \mathrm{H}\right), 7.27-7.23(\mathrm{~m}, \mathrm{ArH}, 1 \mathrm{H}), 7.10-$ $7.07(\mathrm{~m}, \mathrm{ArH}, 1 \mathrm{H}), 6.88(\mathrm{~s}, \mathrm{ArH}, 1 \mathrm{H}), 6.78\left(\mathrm{~d},{ }^{3} J_{\mathrm{HH}}=8.0,1.1 \mathrm{~Hz}\right.$, $\mathrm{ArH}, 1 \mathrm{H}), 4.70\left(\mathrm{~d},{ }^{2} J_{\mathrm{HH}}=10.0 \mathrm{~Hz}, \mathrm{COCH}_{2}, 1 \mathrm{H}\right), 4.04\left(\mathrm{~d},{ }^{2} J_{\mathrm{HH}}=10.0\right.$ $\left.\mathrm{Hz}, \mathrm{COCH}_{2}, 1 \mathrm{H}\right), 2.26$ (s, $\left.\mathrm{CH}_{3}, 3 \mathrm{H}\right), 2.24\left(\mathrm{~s}, \mathrm{CH}_{3}, 3 \mathrm{H}\right), 1.61\left(\mathrm{~s}, \mathrm{CH}_{3}\right.$, $3 \mathrm{H}) .{ }^{13} \mathrm{C}$ NMR $\left(126 \mathrm{MHz}\right.$, DMSO- $\left.d_{6}\right): \delta=170.3(\mathrm{C}=0), 167.5$ $(\mathrm{C}=\mathrm{N}), 142.2(\operatorname{ArC}), 138.9(\operatorname{ArC}), 137.9(\operatorname{ArC}), 137.0(\operatorname{ArC}), 136.2$ (ArC), $134.7(\operatorname{ArC}), 132.3(\operatorname{ArC}), 130.9(\operatorname{ArC}), 130.0(\operatorname{ArC} \times 2)$, 129.6 (ArC $\times 2), 129.5(\operatorname{ArC}), 128.9(\operatorname{ArC} \times 2), 128.7(\operatorname{ArC}), 124.4$ ( $\mathrm{ArC}), 122.1(\mathrm{ArC}), 57.3\left(\mathrm{COCH}_{2}\right), 21.0\left(\mathrm{CH}_{3}\right), 18.5\left(\mathrm{CH}_{3}\right), 17.5$ $\left(\mathrm{CH}_{3}\right)$. ESI-HRMS: $\mathrm{m} / z$ calcd for $\mathrm{C}_{24} \mathrm{H}_{22} \mathrm{~N}_{2} \mathrm{O}[+\mathrm{H}]^{+}$: 355.1805; found: 355.1804 . $\mathrm{LC}-\mathrm{MS}$ purity $(\mathrm{UV})=97 \%, t_{\mathrm{R}}=21.13 \mathrm{~min}$. 1-(2-Bromophenyl)-5-phenyl-1,3-dihydro-2H-1,4-benzodiazepin-2-one (3j)

The reaction was run for $8 \mathrm{~h}$. The product was obtained as white solid (1.00 mmol scale, $31 \mathrm{mg}, 8 \%) .{ }^{1} \mathrm{H}$ NMR (500 MHz, DMSO$\left.d_{6}\right): \delta=7.84\left(\mathrm{~d},{ }^{3} J_{H H}=8.0 \mathrm{~Hz}, \mathrm{ArH}, 1 \mathrm{H}\right), 7.70\left(\mathrm{~d},{ }^{3} J_{\mathrm{HH}}=8.0 \mathrm{~Hz}\right.$, ArH, $1 \mathrm{H}), 7.66-7.59$ ( $\mathrm{m}$, ArH, $4 \mathrm{H})$, 7.52-7.46 (m, ArH, $3 \mathrm{H})$, 7.41-7.37 (m, ArH, $1 \mathrm{H}), 7.32$ (dd, $J=7.8,1.7 \mathrm{~Hz}, \mathrm{ArH}, 1 \mathrm{H}), 7.27$ $\left(\mathrm{d},{ }^{3} J_{H H}=7.0 \mathrm{~Hz}, \mathrm{ArH}, 1 \mathrm{H}\right), 6.92-6.83(\mathrm{~m}, \mathrm{ArH}, 1 \mathrm{H}), 4.69\left(\mathrm{~d},{ }^{2} J_{H H}\right.$ $\left.=10.5 \mathrm{~Hz}, \mathrm{COCH}_{2}, 1 \mathrm{H}\right), 4.01\left(\mathrm{~d},{ }^{2} J_{\mathrm{HH}}=10.5 \mathrm{~Hz}, \mathrm{COCH}_{2}, 1 \mathrm{H}\right) .{ }^{13} \mathrm{C}$ NMR $\left(126 \mathrm{MHz}, \mathrm{CDCl}_{3}\right): \delta=170.7(\mathrm{C}=\mathrm{O}), 168.9(\mathrm{C}=\mathrm{N}), 142.0$ (ArC), $138.9(\operatorname{ArC}), 138.6(\operatorname{ArC}), 134.3(\operatorname{ArC}), 133.7(\operatorname{ArC}), 132.0$ (ArC), $131.0(\operatorname{ArC}), 130.9(\operatorname{ArC}), 130.8(\operatorname{ArC}), 129.9(\operatorname{ArC} \times 2)$, 129.8 (ArC), 129.1 (ArC), $128.8(\operatorname{ArC} \times 2), 124.7(\operatorname{ArC}), 123.0$ (ArC), $121.5(\mathrm{ArC}), 57.0\left(\mathrm{COCH}_{2}\right)$. ESI-HRMS: $\mathrm{m} / z$ calcd for $\mathrm{C}_{21} \mathrm{H}_{15} \mathrm{BrN}_{2} \mathrm{O}[+\mathrm{H}]^{+}:$391.0441; found: 391.0457. LC-MS purity $(\mathrm{UV})=93 \%, t_{\mathrm{R}}=15.23 \mathrm{~min}$.

1-(3'-Trifluoromethylphenyl)-5-phenyl-1,3-dihydro-2H-1,4benzodiazepin-2-one (3k)

The reaction was run for $8 \mathrm{~h}$. The product was obtained as white solid (0.50 mmol scale, $80 \mathrm{mg}, 42 \%) .{ }^{1} \mathrm{H}$ NMR ( $500 \mathrm{MHz}, \mathrm{CDCl}_{3}$ ): $\delta=7.72\left(\mathrm{~d},{ }^{3} J_{\mathrm{HH}}=7.5 \mathrm{~Hz}, \mathrm{ArH}, 2 \mathrm{H}\right), 7.60-7.55(\mathrm{~m}, \mathrm{ArH}, 2 \mathrm{H}), 7.53$ $\left(\mathrm{d},{ }^{3} J_{\mathrm{HH}}=8.5 \mathrm{~Hz}, \mathrm{ArH}, 2 \mathrm{H}\right), 7.49-7.45(\mathrm{~m}, \mathrm{ArH}, 2 \mathrm{H}), 7.44-7.38$ $(\mathrm{m}, \mathrm{ArH}, 3 \mathrm{H}), 7.25-7.20(\mathrm{~m}, \mathrm{ArH}, 1 \mathrm{H}), 6.92\left(\mathrm{~d},{ }^{3} \mathrm{HH}_{\mathrm{H}}=8.5 \mathrm{~Hz}, \mathrm{ArH}\right.$, $1 \mathrm{H}), 4.95\left(\mathrm{~d},{ }^{2} J_{\mathrm{HH}}=10.5 \mathrm{~Hz}, \mathrm{COCH}_{2}, 1 \mathrm{H}\right), 4.02\left(\mathrm{~d},{ }^{2} J_{\mathrm{HH}}=10.5 \mathrm{~Hz}\right.$, $\left.\mathrm{COCH}_{2}, 1 \mathrm{H}\right) .{ }^{13} \mathrm{C}$ NMR $\left(126 \mathrm{MHz}, \mathrm{CDCl}_{3}\right): \delta=170.3(\mathrm{C}=0), 168.3$
$(\mathrm{C}=\mathrm{N}), 143.1(\operatorname{ArC}), 141.3(\operatorname{ArC}), 138.6(\operatorname{ArC}), 132.0\left(\mathrm{q},{ }^{2} \mathrm{JFC}_{\mathrm{FC}}=29.9\right.$ $\mathrm{Hz}, \mathrm{ArC}), 131.9(\operatorname{ArC}), 131.6(\operatorname{ArC}), 131.4(\operatorname{ArC}), 130.7(\operatorname{ArC}), 130.4$ $(\operatorname{ArC}), 129.8(\operatorname{ArC} \times 2), 129.5(\operatorname{ArC}), 128.5(\operatorname{ArC} \times 2), 125.2\left(\mathrm{q}, J_{\mathrm{FC}}=\right.$ $3.5 \mathrm{~Hz}, \operatorname{ArC}), 124.8(\operatorname{ArC}), 124.8(\operatorname{ArC}), 123.5\left(\mathrm{q},{ }^{1} J_{\mathrm{FC}}=273.0 \mathrm{~Hz}\right.$, $\operatorname{ArC}), 124.2\left(\mathrm{q},{ }^{3} J_{\mathrm{FC}}=3.5 \mathrm{~Hz}, \mathrm{ArC}\right), 57.3\left(\mathrm{COCH}_{2}\right)$. ESI-HRMS: $\mathrm{m} / \mathrm{z}$ calcd for $\mathrm{C}_{22} \mathrm{H}_{15} \mathrm{~F}_{3} \mathrm{~N}_{2} \mathrm{O}[+\mathrm{H}]^{+}$: 381.1209; found: 381.1208. LC-MS purity $(U V)=96 \%, t_{R}=21.35 \mathrm{~min}$.

1-Phenyl-5-phenyl-1,3-dihydro-2H-1,4-benzodiazepin-2one (31)

The reaction was run for $8 \mathrm{~h}$. The product was obtained as white solid (0.50 mmol scale, $14 \mathrm{mg}, 9 \%) .{ }^{1} \mathrm{H}$ NMR (500 MHz, $\left.\mathrm{CDCl}_{3}\right)$ : $\delta=7.72\left(\mathrm{~d},{ }^{3} J_{H H}=7.5 \mathrm{~Hz}, \mathrm{ArH}, 2 \mathrm{H}\right), 7.54-7.49$ (m, ArH, $\left.1 \mathrm{H}\right), 7.47$ $\left(\mathrm{d},{ }^{3} J_{H H}=7.5 \mathrm{~Hz}, \mathrm{ArH}, 2 \mathrm{H}\right), 7.43-7.38(\mathrm{~m}, \mathrm{ArH}, 2 \mathrm{H}), 7.37-7.30$ (m, ArH, 2 H), 7.24-7.21 (m, ArH, $3 \mathrm{H}), 7.20-7.16$ (m, ArH, $1 \mathrm{H}$ ), $6.97\left(\mathrm{~d},{ }^{3} J_{H H}=8.5 \mathrm{~Hz}, \mathrm{ArH}, 1 \mathrm{H}\right), 4.96\left(\mathrm{~d},{ }^{2} J_{H H}=10.5 \mathrm{~Hz}, \mathrm{ArH}\right.$, $\left.\mathrm{COCH}_{2}, 1 \mathrm{H}\right), 4.01\left(\mathrm{~d},{ }^{2} \mathrm{~J}_{\mathrm{HH}}=10.5 \mathrm{~Hz}, \mathrm{COCH}_{2}, 1 \mathrm{H}\right) .{ }^{13} \mathrm{C}$ NMR $(126$ $\left.\mathrm{MHz}, \mathrm{CDCl}_{3}\right): \delta=170.7(\mathrm{C}=\mathrm{O}), 168.3(\mathrm{C}=\mathrm{N}), 146.5(\operatorname{ArC}), 143.3$ (ArC), 140.7 (ArC), $138.6(\operatorname{ArC}), 131.3(\operatorname{ArC}), 130.7(\operatorname{ArC}), 130.3$ (ArC), $129.6(\operatorname{ArC} \times 2), 129.3(\operatorname{ArC} \times 2), 128.4(\operatorname{ArC} \times 2), 128.3(\operatorname{ArC}$ $\times 2), 127.5(\mathrm{ArC}), 124.7(\mathrm{ArC}), 124.2(\mathrm{ArC}), 57.2\left(\mathrm{COCH}_{2}\right)$. ESIHRMS: $m / z$ calcd for $\mathrm{C}_{21} \mathrm{H}_{16} \mathrm{~N}_{2} \mathrm{O}[+\mathrm{H}]^{+}:$313.1335; found: 313.1338. LC-MS purity (UV) $=90 \%, t_{\mathrm{R}}=16.10 \mathrm{~min}$.

1-(4-Nitrophenyl)-3-(4-nitrophenyl)-5-phenyl-1,3-dihydro2H-1,4-benzodiazepin-2-one (7)

The product was obtained as white solid $(0.60 \mathrm{mmol}$ scale, 2 equiv of diaryliodonium triflate, $146 \mathrm{mg}, 51 \%$ ). ${ }^{1} \mathrm{H}$ NMR (500 $\left.\mathrm{MHz}, \mathrm{CDCl}_{3}\right): \delta=8.34-8.22(\mathrm{~m}, \mathrm{ArH}, 4 \mathrm{H}), 7.85-7.79(\mathrm{~m}, \mathrm{ArH}, 2$ $\mathrm{H})$, 7.77-7.71 (m, ArH, $2 \mathrm{H}), 7.74-7.57$ (m, ArH, $3 \mathrm{H})$, 7.59-7.50 (m, ArH, $3 \mathrm{H}), 7.41-7.32(\mathrm{~m}, \mathrm{ArH}, 2 \mathrm{H}), 7.07-7.02(\mathrm{~m}, \mathrm{ArH}, 1 \mathrm{H})$. ${ }^{13} \mathrm{C}$ NMR $\left(126 \mathrm{MHz}, \mathrm{CDCl}_{3}\right): \delta=166.0(\mathrm{C}=\mathrm{O}), 158.3(\mathrm{C}=\mathrm{N}), 149.1$ (ArC), 146.7 ( $\operatorname{ArC}), 145.6(\operatorname{ArC}), 144.1(\operatorname{ArC}), 143.3(\operatorname{ArC}), 135.0$ (ArC), 132.6 (ArC), $131.5(\operatorname{ArC}), 129.9(\operatorname{ArC}), 129.6(\operatorname{ArC}), 129.5$ $(\operatorname{ArC} \times 2), 128.9(\operatorname{ArC} \times 2), 126.8(\operatorname{ArC} \times 2), 126.3(\operatorname{ArC}), 125.4$ $(\mathrm{ArC}) 124.5(\mathrm{ArC} \times 2), 124.3(\mathrm{ArC} \times 2), 121.3(\mathrm{ArC} \times 2)$. ESI-HRMS: $\mathrm{m} / \mathrm{z}$ calcd for $\mathrm{C}_{26} \mathrm{H}_{17} \mathrm{~N}_{5} \mathrm{O}_{5}[+\mathrm{H}]^{+}: 480.1230$; found: 480.1245. LCMS purity $(\mathrm{UV})=95 \%, t_{\mathrm{R}}=18.35 \mathrm{~min}$.

\section{1,1'-Oxybis(4-nitrobenzene)}

To a solution of (4-nitrophenyl)phenyliodonium triflate $(30 \mathrm{mg}$, $0.06 \mathrm{mmol}$ ) in DCE $(1 \mathrm{~mL}$ ) was added sodium hydroxide (aq., 1 $\mathrm{mL}$ ) and stirred for $20 \mathrm{~min}$ at room temperature. Upon completion, the reaction was diluted with dichloromethane $(5 \mathrm{~mL} \times 3)$ and the layers were separated. Combined organic layers were dried $\left(\mathrm{MgSO}_{4}\right)$ and concentrated under reduced pressure to afford the product as a white powder ( $7 \mathrm{mg}, 43 \%) .{ }^{1} \mathrm{H}$ NMR (500 $\left.\mathrm{MHz}, \mathrm{CDCl}_{3}\right): \delta=8.33-8.27(\mathrm{~m}, \mathrm{ArH}, 4 \mathrm{H}), 7.19-7.14(\mathrm{~m}, \mathrm{ArH}, 4$ H) ${ }^{13}{ }^{C}$ NMR $\left(126 \mathrm{MHz}, \mathrm{CDCl}_{3}\right): \delta=160.6(\operatorname{ArC} \times 2), 144.2(\operatorname{ArC} \times$ $2), 126.2(\mathrm{ArC} \times 4), 119.3(\mathrm{ArC} \times 4)$. ESI-HRMS: $m / z$ calcd for $\mathrm{C}_{12} \mathrm{H}_{8} \mathrm{~N}_{2} \mathrm{O}_{5}$ [+H $]^{+}:$261.0511; found: 261.0513 .

(43) CCDC numbers 1560492-1560494 contain the supplementary crystallographic data for compounds $\mathbf{3 a}, \mathbf{3 h}, \mathbf{3 i}$. The data can be obtained free of charge from The Cambridge Crystallographic Data Centre via www.ccdc.cam.ac.uk/getstructures. 
R. Khan et al.

THIEME

OPEN

